\title{
Association of six-minute walk test distance with postoperative complications in non-cardiac surgery: a secondary analysis of a multicentre prospective cohort study
}

\section{Association entre la distance parcourue pendant le test de marche de six minutes et les complications postopératoires en chirurgie non cardiaque : une analyse secondaire d'une étude de cohorte prospective multicentrique}

\author{
Ryan J. Ramos, BSc $\cdot$ Karim S. Ladha, MD, MSc $\cdot$ Brian H. Cuthbertson, MBChB, MD $\cdot$ \\ Mark A. Shulman, MBBS, MPH • Paul S. Myles, MBBS, MPH, MD, DSc • \\ Duminda N. Wijeysundera, MD, PhD, FAHA (i) on behalf of the METS Study \\ Investigators
}

Received: 9 September 2020/Revised: 2 October 2020/Accepted: 5 October 2020/Published online: 13 January 2021

(c) The Author(s) 2021

\begin{abstract}
Purpose The six-minute walk test (6MWT) is a simple and valid test for assessing cardiopulmonary fitness. Nevertheless, the relationship between preoperative 6MWT distance and postoperative complications is uncertain. We conducted a secondary analysis of the 6MWT nested cohort substudy of the Measurement of Exercise Tolerance before Surgery study to determine if
\end{abstract}

Supplementary Information The online version contains supplementary material available at https://doi.org/10.1007/s12630020-01909-9.

\section{R. J. Ramos, BSc}

Faculty of Medicine, University of Toronto, Toronto, ON, Canada

K. S. Ladha, MD, MSc - D. N. Wijeysundera, MD, PhD, FAHA $(\square)$

Department of Anesthesia, St Michael's Hospital, 30 Bond

Street, Toronto, ON M5B 1W8, Canada

e-mail: d.wijeysundera@utoronto.ca

Li Ka Shing Knowledge Institute, St Michael's Hospital, Toronto, ON, Canada

Department of Anesthesiology and Pain Medicine, University of Toronto, Toronto, ON, Canada
6MWT distance predicts postoperative complications or death.

Methods This analysis included 545 adults ( $\geq 40 \mathrm{yr}$ ) who were at elevated cardiac risk and had elective inpatient non-cardiac surgery at 15 hospitals in Canada, Australia, and New Zealand. Each participant performed a preoperative 6MWT and was followed for 30 days after surgery. The primary outcome was moderate or severe inhospital complications. The secondary outcome was 30-day death or myocardial injury. Multivariable logistic regression modelling was used to characterize the

\section{B. H. Cuthbertson, $\mathrm{MBChB}, \mathrm{MD}$}

Department of Anesthesiology and Pain Medicine, University of Toronto, Toronto, ON, Canada

Institute of Health Policy, Management and Evaluation,

University of Toronto, Toronto, ON, Canada

Department of Critical Care Medicine, Sunnybrook Health Sciences Centres, Toronto, ON, Canada

M. A. Shulman, MBBS, MPH - P. S. Myles, MBBS, MPH, MD, DSc

Department of Anaesthesia and Perioperative Medicine, Alfred Hospital and Monash University, Melbourne, VIC, Australia 
adjusted association of 6MWT distance with these outcomes.

Results Seven participants (1\%) terminated their 6MWT sessions early because of lower limb pain, dyspnea, or dizziness. Eighty-one (15\%) participants experienced moderate or severe complications and 69 (13\%) experienced 30-day myocardial injury or death. Decreased 6MWT distance was associated with increased odds of moderate or severe complications (adjusted odds ratio, 1.32 per $100 \mathrm{~m}$ decrease; 95\% confidence interval, 1.01 to $1.73 ; P=0.045)$. There was no association of 6MWT distance with myocardial injury or 30-day death (non-linear association; $P=0.49$ ).

Conclusion Preoperative 6MWT distance had a modest association with moderate or severe complications after inpatient non-cardiac surgery. Further studies are needed to determine the optimal role of the 6MWT as an objective exercise test for informing preoperative risk stratification.

\section{Résumé}

Objectif Le test de marche de six minutes (6MWT) est un test simple et validé pour évaluer la santé cardiopulmonaire. Néanmoins, la relation entre la distance parcourue lors d'un 6MWT préopératoire et les complications postopératoires est inconnue. Nous avons effectué une analyse secondaire de la sous-étude de cohorte imbriquée du 6MWT dans l'étude de la Mesure de la tolérance à l'exercice avant chirurgie (Measurement of Exercise Tolerance before Surgery) afin de déterminer si la distance parcourue lors du 6MWT était prédictive de complications postopératoires ou de décès.

Méthode Cette analyse comprenait 545 adultes $(\geq 40$ ans) courant un risque cardiaque élevé et hospitalisés pour une chirurgie non cardiaque élective dans 15 hôpitaux au Canada, en Australie et en Nouvelle-Zélande. Chaque participant a exécuté un $6 M W T$ préopératoire et a été suivi pendant 30 jours post chirurgie. Le critère d'évaluation principal touchait aux complications modérées ou graves à l'hôpital. Le critère secondaire était le décès à 30 jours ou une lésion myocardique. Un modèle de régression logistique multivariée a été employé pour caractériser l'association ajustée entre la distance parcourue lors du $6 M W T$ et ces critères d'évaluation.

Résultats Sept participants (1\%) n'ont pas terminé leurs séances de 6MWT en raison de douleurs aux membres inférieurs, de dyspnée ou de vertiges. Quatre-vingt-un (15 $\%)$ participants ont souffert de complications modérées ou graves et 69 (13\%) ont subi une lésion myocardique ou sont décédés à 30 jours. La diminution de la distance parcourue au 6MWT a été associée à une augmentation du risque de complications modérées ou graves (rapport de cotes ajusté, 1,32 par diminution de $100 \mathrm{~m}$; intervalle de confiance $95 \%, 1,01$ à 1,73; $P=0,045)$. Aucune association n'a été observée entre la distance parcourue au 6MWT et la lésion myocardique ou le décès à 30 jours (association non linéaire; $P=0,49$ ).

Conclusion La distance parcourue lors d'un 6MWT préopératoire a été modestement associée à des complications modérées ou graves après une chirurgie non cardiaque avec hospitalisation. D'autres études sont nécessaires pour déterminer le rôle optimal du 6MWT en tant que test d'exercice objectif pour informer la stratification préopératoire des risques.

Keywords preoperative risk assessment . 6-minute walk test - postoperative complications . functional capacity

Assessment of functional capacity is a ubiquitous component of preoperative evaluation for major surgery. A key intended purpose for evaluating functional capacity is to identify patients at increased risk of major postoperative complications. The most common method for assessing functional capacity is "subjective assessment", where a physician makes a judgement following an unstructured patient interview. Recent research has shown that subjective assessment is neither an accurate measure of preoperative cardiopulmonary fitness nor an important prognostic indicator of perioperative risk. ${ }^{1}$ A possibly better alternative for assessing functional capacity is cardiopulmonary exercise testing (CPET), which has been described as the "goldstandard" non-invasive measurement of the integrated cardiopulmonary response to exercise. ${ }^{2}$ Metrics derived from CPET, including peak oxygen consumption, anaerobic threshold, and inability to complete the exercise test have moderate ability to predict major postoperative complications. ${ }^{1,3,4}$ Despite these promising data, an important practical impediment to widespread adoption of preoperative CPET is the associated need for specialized equipment and trained personnel.

A more feasible alternative may be the six-minute walk test (6MWT), which is a simple, cheap, and clinically acceptable measure of cardiopulmonary fitness. ${ }^{5}$ The 6MWT involves walking for six minutes on a flat 30-m course while standardized instructions and encouragement are provided. ${ }^{5}$ Performance is based on the total distance walked. The 6MWT has excellent reliability, and good construct validity based on moderate-to-strong correlation to CPET performance. ${ }^{6}$ The test is safe, with significant adverse events being rare $(1 \%){ }^{6}$ The $6 \mathrm{MWT}$ has been considered a possible tool for preoperative assessment of functional capacity. In limited prior research, it was a similarly valid measure of cardiopulmonary fitness in 
surgical patients, based on moderate correlation with peak oxygen consumption and anaerobic threshold measured by CPET. $^{7}$ Nonetheless, there remains uncertainty as to whether 6MWT distance predicts complications after major non-cardiac surgery. Outside the specialized context of lung cancer resection surgery, ${ }^{8,9}$ prior relevant research consists of small studies ( $<150$ participants) with mixed results. Some studies found an association between 6MWT distance and complications, ${ }^{10-15}$ while others did not. ${ }^{16,17}$ Thus, there is a need for larger high-quality studies to better assess the role, if any, of the 6MWT in preoperative risk stratification.

We therefore conducted a secondary analysis of the 6MWT substudy of the Measurement of Exercise Tolerance before Surgery (METS) prospective cohort study. The METS study was a multicentre prospective cohort study that compared subjective assessment, CPET, natriuretic peptides, and a structured questionnaire for predicting death and major complications in 1,400 patients undergoing major non-cardiac surgery. ${ }^{1}$ The 6MWTMETS substudy included 545 participants who underwent both 6MWT and CPET before elective inpatient noncardiac surgery. Its primary aim was to characterize the association of 6MWT distance with two validated patientreported outcomes: 30-day quality of recovery and oneyear disability-free survival. ${ }^{18}$ In this secondary analysis of the 6MWT-METS substudy, we sought to determine whether 6MWT distance predicted two clinically relevant postoperative adverse events (i.e., moderate or severe inhospital postoperative complications, myocardial injury, or 30-day postoperative death), and to characterize the association between preoperative 6MWT distance and CPET performance.

\section{Methods}

Study design

We conducted a secondary analysis of the 6MWT-METS substudy. The METS study was an international multicentre prospective cohort study of adults ( $\geq 40 \mathrm{yr}$ ) who were deemed to be at elevated risk for postoperative cardiac complications and scheduled to have major elective inpatient non-cardiac surgery. The objectives, methods, and primary results of the METS study have been previously reported. ${ }^{1,19}$ The 6MWT-METS substudy was a nested cohort study at 15 hospitals in Canada, Australia, and New Zealand. Each study centre obtained research ethics board approval before commencing recruitment, and all participants provided written informed consent. ${ }^{18}$ Additional research ethics approval for this secondary analysis was provided by the Unity Health Toronto Research Ethics Board.

Participants and study procedures

Participants were eligible for inclusion in the METS study if they were aged $\geq 40 \mathrm{yr}$, scheduled to have elective inpatient non-cardiac surgery, and had one or more risk factors for ischemic heart disease or postoperative cardiac complications (Appendix 1). Exclusion criteria are presented on Appendix 2. Prior to surgery, all participants underwent CPET on a cycle ergometer and underwent blood sampling to measure serum N-terminal pro-B-type natriuretic peptide (NT pro-BNP) concentrations. At the 15 hospitals participating in the substudy, all participants in the main METS study were approached to consider participation in the 6MWT-METS substudy. Participants who consented to the substudy also completed the 6MWT before surgery. Participants were followed for 30 days after surgery to ascertain mortality and specific complications (Appendix 3).

\section{Six-minute walk test and CPET procedures}

The 6MWT was conducted according to the American Thoracic Society guidelines. ${ }^{20}$ Where possible, the 6MWT was performed on a different day from CPET; otherwise, the 6MWT was completed after CPET, with a minimum two-hour break between tests. After delivery of standardized instructions, participants completed as many laps as possible in six minutes on a flat indoor 30-m course. Walk distance $(\mathrm{m})$ was calculated from the number of complete laps and distance walked in any final partial lap. This distance represents a valid test performance, regardless of the occurrence of rests or early termination. Healthcare providers were kept blinded to the 6MWT results.

Participants performed symptom-limited incremental CPET on a computer-controlled, electromagnetically braked cycle ergometer using a standardized protocol. A trained investigator at each centre used full-page graphs of the plotted local CPET data to determine peak oxygen consumption and anaerobic threshold using standardized protocols. The protocols used for the conduct and interpretation of CPET have been previously described. ${ }^{1,19}$

\section{Outcomes}

The primary outcome was postoperative moderate or severe in-hospital complications (including fatal events). The severity of complications was graded using a modified Clavien-Dindo classification system (Appendix 3). ${ }^{21,22}$ The secondary outcome was myocardial injury or death 
within 30 days after surgery. Myocardial injury was defined as a troponin concentration exceeding both the $99^{\text {th }}$ percentile upper reference limit and threshold at which the assay coefficient of variation was $10 \% .^{23}$ Participants underwent daily electrocardiogram and troponin measurements up to the third day after surgery or hospital discharge (whichever came first). Research personnel ascertaining the presence of complications were blinded to CPET and 6MWT results.

\section{Statistical analysis}

All participants who completed the 6MWT and had surgery were included in the main analyses. Descriptive statistics were initially used to characterize the sample with respect to baseline characteristics, operative features, and postoperative outcomes, both overall and across strata defined by tertiles of 6MWT distance. Categorical variables were compared using Chi square or Fisher's exact test, while continuous variables were compared using analysis of variance or the Kruskal-Wallis test.

We then applied bivariate tests to compare strata defined by the presence or absence of the primary and secondary outcomes. Categorical data were compared using the Chi square or Fisher exact test, while continuous variables were compared using the two-sample $t$ test or Mann-Whitney U test. Multivariable logistic regression models were used to separately evaluate the adjusted association of preoperative 6MWT distance with these outcomes. Model covariates were selected a priori based on clinical sensibility, prior evidence, their inclusion in guideline-recommended assessment algorithms (i.e., Revised Cardiac Risk Index [RCRI] and natriuretic peptides for cardiac risk stratification), consistency with prior analyses of the METS study cohort, ${ }^{1}$ and the need to prevent model overfitting. For the model predicting moderate or severe complications, the covariates were age, sex, and high-risk surgery (intraperitoneal, retroperitoneal, intrathoracic, or major vascular procedures). ${ }^{1,24}$ For the model predicting 30-day death or myocardial injury, the covariates were age, sex, RCRI score, and NT pro-BNP concentration. ${ }^{1,24}$ As recommended by the Canadian Cardiovascular Society guidelines, NT pro-BNP concentration was dichotomized based on a $300 \mathrm{ng} \cdot \mathrm{L}^{-1}$ threshold. ${ }^{25}$ To account for nonlinear relationships between continuous predictor variables (i.e., 6MWT distance, age) and outcomes, we used restricted cubic spline plots to determine which transformation or categorization-if any-was necessary. Associations were expressed as adjusted odds ratios (aORs) with $95 \%$ confidence intervals (CIs). Model discrimination was characterized by the c-index, while calibration was assessed using the Hosmer-Lemeshow statistic. If a regression model showed preoperative 6MWT distance to be associated with the outcome of interest, the additional prognostic information associated with 6MWT distance was characterized by the change in the area under the receiver-operating-characteristic (ROC) curve, ${ }^{26}$ continuous net reclassification index (NRI), and integrated discrimination improvement (IDI). ${ }^{27,28}$

The primary analysis was a complete case analysis because missing data were uncommon $(<6 \%)$ and largely pertained to information on baseline characteristics. We assumed that the single participant lost to follow-up between hospital discharge and the $30^{\text {th }}$ postoperative day remained alive during this follow-up window. Analyses were conducted using the $\mathrm{R}$ statistical language (Version 4.0.2; R Foundation for Statistical Computing, Vienna, Austria) and RStudio (Version 1.3.1056; RStudio PBC, Boston, MA, USA) software. Statistical significance was defined as a two-tailed $P$ value $<0.05$ and no adjustment was made for multiple comparisons. The number of participants available for this substudy was determined by the sample size calculations for the METS study and 6MWT-METS substudy. ${ }^{1,18,19}$

\section{Cardiopulmonary exercise testing subset analysis}

To determine the correlation between 6MWT distance and measures of cardiopulmonary fitness from CPET (i.e., peak oxygen consumption, anaerobic threshold), we conducted a subset analysis of participants who underwent both 6MWT and CPET. This analysis was restricted to patients who successfully completed their exercise test (6MWT $v s$ peak oxygen consumption) or who had a measurable anaerobic threshold (6MWT $v s$ anaerobic threshold) because peak oxygen consumption is unlikely to represent cardiopulmonary fitness when an exercise test is terminated early for reasons (i.e., arthritis pain) other than the patient reaching their limit of tolerance, and about $9 \%$ of patients in the main METS study sample had an indeterminate anaerobic threshold. ${ }^{1}$ The correlation between 6MWT distance and CPET measures was characterized using the Spearman coefficient.

\section{Sensitivity analyses}

We repeated the analyses after including different model covariates and using bootstrap resampling (1,000 samples) to internally validate the regression models.

Post hoc analyses

In response to comments from external peer reviewers, we conducted a post hoc analysis to determine whether performance of preoperative CPET and the 6MWT on the same day altered the prognostic accuracy of 6MWT 
distance. First, mean 6MWT distance was compared between participants who performed the 6MWT on the same day as CPET compared with participants who did not. Second, we evaluated whether performance of CPET on the same day as the 6MWT modified the adjusted association between 6MWT distance and the primary outcome (i.e., moderate or severe complications). A multivariable logistic regression model was estimated using the following predictor variables: age, sex, highrisk surgery, 6MWT distance, same-day CPET testing, and the interaction between 6MWT distance and same-day CPET testing. Effect modification was assessed based on the statistical significance of the interaction term.

\section{Results}

Participant characteristics

From 31 July 2013 to 16 December 2015, 574 patients were recruited into the 6MWT-METS substudy (Fig. 1). All participants performed the 6MWT before surgery while $545(95 \%)$ had surgery. The 6MWT was terminated early in seven participants (1.2\%) because of dyspnea, dizziness, and lower limb pain. The sample characteristics, both overall and across tertiles of 6MWT performance, are presented in Table 1 . The overall mean (standard deviation [SD]) age was $64(11) \mathrm{yr}$, with $39 \%(n=215)$ being female,
Fig. 1 Patient flow diagram showing the number of patients included in the analysis. 6MWT = 6-minute walk test; NT pro$\mathrm{BNP}=\mathrm{N}$-terminal pro-B-type natriuretic peptide

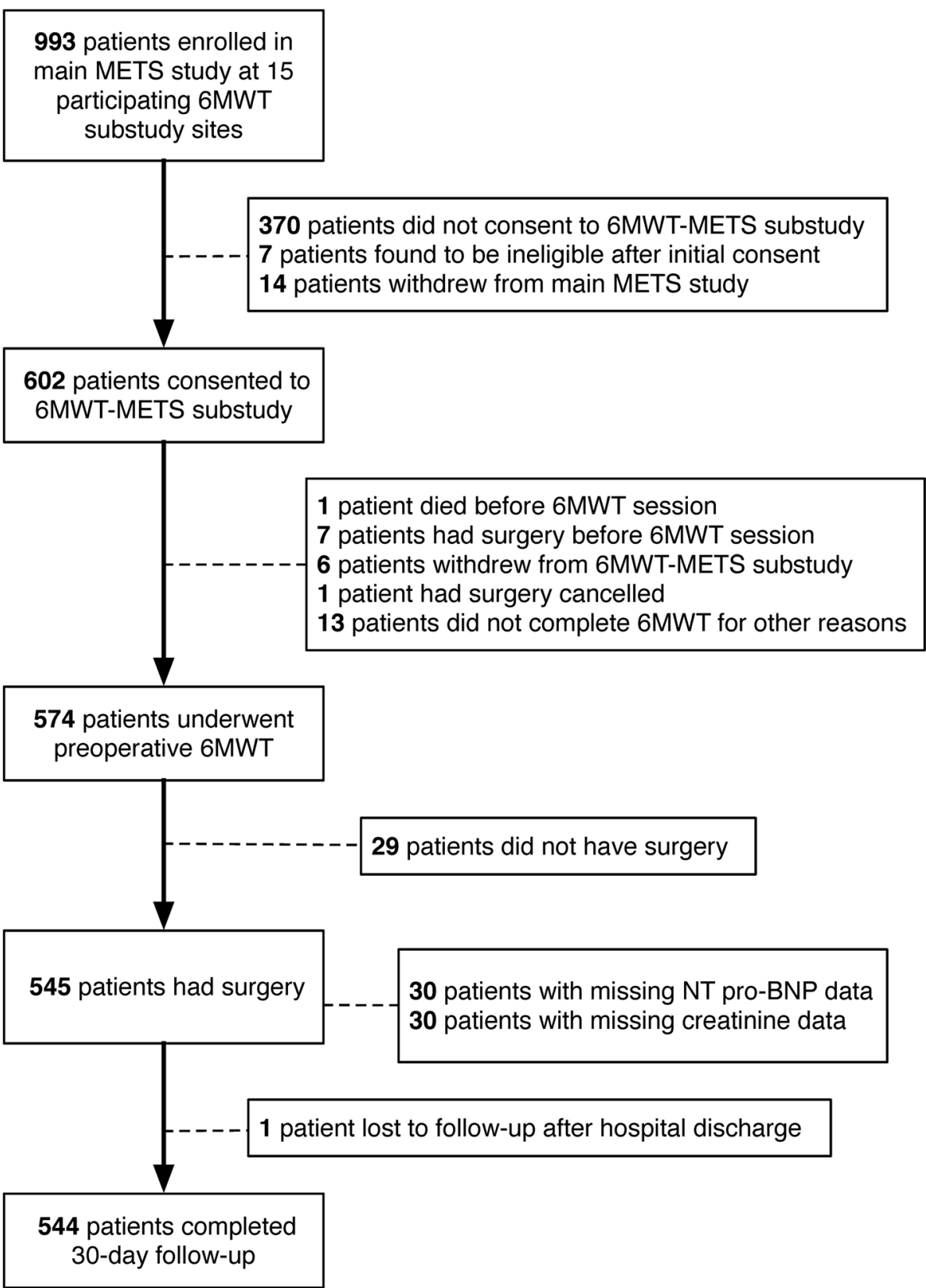


Table 1 Patient characteristics $(n=545)$ across 6MWT distance tertiles

\begin{tabular}{|c|c|c|c|c|c|}
\hline Variable & Low $(<435 \mathrm{~m})$ & Middle & $\operatorname{High}(>510 \mathrm{~m})$ & Total & $P$ value \\
\hline \multicolumn{6}{|l|}{ Patient characteristics } \\
\hline Age $(y r)$, mean $(S D)$ & $68(10)$ & $63(11)$ & $61(10)$ & $64(11)$ & $<0.001$ \\
\hline Female sex, $n(\%)$ & $90(50)$ & $72(38)$ & $53(30)$ & 215 (39) & $<0.001$ \\
\hline BMI $\left(\mathrm{kg} \cdot \mathrm{m}^{-2}\right)$, mean $(\mathrm{SD})$ & $31(8)$ & $30(7)$ & $29(8)$ & $30(7)$ & 0.08 \\
\hline \multicolumn{6}{|l|}{ Comorbidities, $n(\%)$} \\
\hline Coronary artery disease & $41(23)$ & $34(18)$ & $27(15)$ & $102(19)$ & 0.16 \\
\hline Heart failure & $11(6)$ & $4(2)$ & $3(2)$ & $18(3)$ & 0.03 \\
\hline Cerebrovascular disease & $14(8)$ & $8(4)$ & $9(5)$ & $31(6)$ & 0.31 \\
\hline Peripheral artery disease & $10(6)$ & $7(4)$ & $2(1)$ & $19(4)$ & 0.07 \\
\hline Diabetes mellitus & $46(26)$ & $38(20)$ & $28(16)$ & $112(21)$ & 0.06 \\
\hline Hypertension & $124(69)$ & $124(66)$ & $95(53)$ & $343(63)$ & 0.003 \\
\hline Atrial fibrillation & $15(8)$ & $5(3)$ & $5(3)$ & $25(5)$ & 0.01 \\
\hline Recent smoker* & $22(12)$ & $42(23)$ & 30 (17) & 94 (17) & 0.04 \\
\hline Obstructive lung disease $^{\dagger}$ & $35(20)$ & $24(13)$ & $18(10)$ & $77(14)$ & 0.03 \\
\hline Arthritis & $80(47)$ & $52(30)$ & $39(23)$ & $171(31)$ & $<0.001$ \\
\hline \multicolumn{6}{|l|}{ Renal function, $(n=515), n(\%) \ddagger$} \\
\hline $\mathrm{eGFR} \geq 60 \mathrm{~mL} \cdot \mathrm{min}^{-1}(1.73 \mathrm{~m})^{-2}$ & $139(81)$ & $157(90)$ & $159(94)$ & $455(88)$ & 0.005 \\
\hline eGFR $30-59 \mathrm{~mL} \cdot \min ^{-1}(1.73 \mathrm{~m})^{-2}$ & $29(17)$ & $17(9)$ & $8(5)$ & $54(11)$ & \\
\hline $\mathrm{eGFR}<30 \mathrm{~mL} \cdot \mathrm{min}^{-1}(1.73 \mathrm{~m})^{-2}$ or dialysis & $3(2)$ & $1(1)$ & $2(1)$ & $6(1)$ & \\
\hline Peak oxygen consumption $\left(\mathrm{mL} \mathrm{O}_{2} \mathrm{~kg}^{-1} \min ^{-1}\right)(n=431)$, mean (SD) & $18(6)$ & $21(7)$ & $24(7)$ & $21(7)$ & $<0.001$ \\
\hline Anaerobic threshold $\left(\mathrm{mL} \mathrm{O}_{2} \mathrm{~kg}^{-1} \min ^{-1}\right)(\mathrm{n}=420)$, mean $(\mathrm{SD})$ & $12(4)$ & $13(5)$ & $15(4)$ & $14(4)$ & $<0.001$ \\
\hline \multicolumn{6}{|l|}{ Natriuretic peptide concentration $(n=515), n(\%)$} \\
\hline NT pro-BNP $<100 \mathrm{ng} \cdot \mathrm{L}^{-1}$ & $71(41)$ & $88(50)$ & $111(66)$ & $270(52)$ & $<0.001$ \\
\hline NT pro-BNP $100 \mathrm{ng} \cdot \mathrm{L}^{-1}$ to $<300 \mathrm{ng} \cdot \mathrm{L}^{-1}$ & $54(32)$ & $67(38)$ & $42(25)$ & $163(32)$ & \\
\hline NT pro-BNP $\mathrm{ng} \cdot \mathrm{L}^{-1} \geq 300 \mathrm{ng} \cdot \mathrm{L}^{-1}$ & $46(27)$ & $20(12)$ & $16(9)$ & $82(16)$ & \\
\hline \multicolumn{6}{|l|}{ ASA physical status score, $n(\%)$} \\
\hline I & $5(3)$ & $7(4)$ & $10(6)$ & $22(4)$ & 0.06 \\
\hline II & $88(49)$ & $109(58)$ & $110(61)$ & $307(56)$ & \\
\hline III & $81(45)$ & $70(37)$ & $57(32)$ & $208(38)$ & \\
\hline IV & $5(3)$ & $1(1)$ & $2(1)$ & $8(2)$ & \\
\hline \multicolumn{6}{|l|}{ Revised Cardiac Risk Index $(n=518), n(\%)$} \\
\hline Class 1 & $56(33)$ & $56(32)$ & $55(32)$ & $167(32)$ & 0.28 \\
\hline Class 2 & $66(39)$ & $82(46)$ & $84(49)$ & $232(45)$ & \\
\hline Class 3 & $40(23)$ & $28(16)$ & $27(16)$ & $95(18)$ & \\
\hline Class 4 & $9(5)$ & $10(6)$ & $5(3)$ & $24(5)$ & \\
\hline \multicolumn{6}{|l|}{ Operative characteristics } \\
\hline \multicolumn{6}{|l|}{ Procedure type, $n(\%)$} \\
\hline Vascular & $8(5)$ & $7(4)$ & $2(1)$ & $17(3)$ & $<0.001$ \\
\hline Intrathoracic & $6(3)$ & $7(4)$ & $9(5)$ & $22(4)$ & \\
\hline Intraperitoneal or retroperitoneal & $52(29)$ & $74(39)$ & $53(30)$ & $179(33)$ & \\
\hline Urological or gynecological & $37(21)$ & $48(26)$ & $72(40)$ & $157(29)$ & \\
\hline Head-and-neck & $18(10)$ & $24(13)$ & $15(8)$ & $57(11)$ & \\
\hline Orthopedic & $54(30)$ & $19(10)$ & $15(8)$ & $88(16)$ & \\
\hline Other & $4(2)$ & $8(4)$ & $13(7)$ & $25(4)$ & \\
\hline
\end{tabular}


Table 1 continued

\begin{tabular}{|c|c|c|c|c|c|}
\hline Variable & Low $(<435 \mathrm{~m})$ & Middle & $\operatorname{High}(>510 \mathrm{~m})$ & Total & $P$ value \\
\hline \multicolumn{6}{|l|}{ Postoperative hospitalization } \\
\hline Postoperative length-of-stay (day), median [IQR] & $4[2-7]$ & $4[2-6]$ & $4[2-12]$ & $4[2-6]$ & 0.56 \\
\hline \multicolumn{6}{|c|}{$\begin{array}{l}\text { Data displayed are for lower }(<435 \mathrm{~m}) \text {, middle and upper }(>510 \mathrm{~m}) \text { tertiles of 6MWT distances. Categorical variables were compared using } \chi^{2} \\
\text { or Fisher's exact test, while continuous variables were compared using analysis of variance (ANOVA) or the Kruskal-Wallis test }\end{array}$} \\
\hline \multicolumn{6}{|c|}{$\begin{array}{l}\mathrm{ASA}=\text { American Society of Anesthesiologists; } \mathrm{BMI}=\text { body mass index } ; \text { eGFR }=\text { estimated glomerular filtration rate; } \mathrm{IQR}=\text { interquartile range; } \\
6 \mathrm{MWT}=\text { six-minute walk test; NT pro-BNP }=\mathrm{N} \text {-terminal pro-B-type natriuretic peptide }\end{array}$} \\
\hline \multicolumn{6}{|l|}{ *Current smoker or quit within the previous year } \\
\hline \multicolumn{6}{|c|}{${ }^{\dagger}$ Prior diagnosis of asthma, reactive airways disease, chronic obstructive lung disease, chronic bronchitis, or emphysema } \\
\hline \multicolumn{6}{|c|}{$\begin{array}{l}\text { Estimated glomerular filtration rate was calculated using the preoperative serum creatinine concentration and Chronic Kidney Disease } \\
\text { Epidemiology Collaboration equation }\end{array}$} \\
\hline
\end{tabular}

$62 \%(n=336)$ having major abdominal or pelvic procedures, $63 \%(n=327)$ being classified as RCRI class 2 or 3 , and $16 \%(n=82)$ having preoperative NT pro-BNP concentrations exceeding the high-risk threshold of 300 ng. $\mathrm{L}^{-1}$. Five hundred and twenty-three $(96 \%)$ patients had non-thoracic surgery. The mean (SD) preoperative 6MWT distance was $474(100) \mathrm{m}$. When tertiles of 6MWT distance were compared, patients in the lower tertiles were more likely to be older and female, as well as more likely to have some comorbidities (e.g., arthritis, obstructive lung disease, renal insufficiency) and elevated NT pro-BNP concentrations.

\section{Postoperative outcomes}

Eighty-one (15\%) participants experienced moderate or severe complications and 69 (13\%) experienced myocardial injury or 30-day death. Among the 81 participants experiencing the primary outcome, common individual events included major bleeding (26\%), reoperation (20\%), surgical site infection (19\%), and pneumonia (14\%) (eTable 1 in the Electronic Supplementary Material [ESM]). Individual events associated with 30-day death or myocardial injury are presented in eTable 2 (ESM).

Prediction of moderate or severe postoperative complications

In unadjusted comparisons, participants who did or did not experience moderate or severe complications differed with respect to cardiovascular comorbidities (i.e., atrial fibrillation), NT pro-BNP concentration, RCRI score, and surgery type (Table 2). Mean (SD) 6MWT distance was 457 (97) $\mathrm{m}$ in patients who experienced moderate or severe complications compared with 477 (100) $\mathrm{m}$ in those who did not $(P=0.10)$.

Restricted cubic spline analyses showed a linear adjusted association between 6MWT distance and complications (eFig. 1, ESM). We therefore considered 6MWT distance a continuous variable in the final multivariable regression model, where decreased 6MWT distance was associated with increased adjusted odds of postoperative complications (aOR, 1.32 per $100 \mathrm{~m}$ decrease; 95\% CI, 1.01 to $1.73 ; P=0.045$ ) (Table 3 ). Preoperative 6MWT distance did not provide significant additional prognostic accuracy on top of age, sex, and highrisk surgery based on the change in the area under the ROC curve (increase in area from 0.67 to $0.68 ; P=0.42$ ), continuous NRI $(0.18 ; 95 \% \mathrm{CI},-0.06$ to $0.41 ; P=0.14)$ and IDI $(0.008 ; 95 \% \mathrm{CI},-0.0003$ to $0.017 ; P=0.06)$.

Prediction of 30-day death or myocardial injury

In unadjusted comparisons, the participants who did or did not experience myocardial injury or 30-day death or differed with respect to age, cardiovascular comorbidities (i.e., coronary artery disease, cerebrovascular disease), NT pro-BNP concentration, RCRI, surgery type, and 6MWT distance (eTable 3, ESM). After adjustment for RCRI score and NT pro-BNP concentration, restricted cubic spline analyses showed a non-linear association between 6MWT distance and myocardial injury or 30-day death (eFig. 2, ESM). In multivariable logistic regression analyses, the 6WMT distance was therefore modelled flexibly using a restricted cubic spline function. There was no strong association of 6MWT distance with 30-day death or myocardial injury $(P=0.49)$ (Table 4$)$. 
Table 2 Bivariate comparisons between patients who did $v s$ did not experience postoperative in-hospital moderate or severe complications

\begin{tabular}{|c|c|c|c|}
\hline Variable & $\begin{array}{l}\text { Mild or no complications } \\
(n=464)\end{array}$ & $\begin{array}{l}\text { Moderate or severe complications } \\
(n=81)\end{array}$ & $P$ value \\
\hline \multicolumn{4}{|l|}{ Patient characteristics } \\
\hline Age (yr), mean (SD) & $64(11)$ & $65(11)$ & 0.2 \\
\hline Female sex, $n(\%)$ & $191(41)$ & $24(30)$ & 0.05 \\
\hline BMI $\left(\mathrm{kg} \cdot \mathrm{m}^{-2}\right)$, mean $(\mathrm{SD})$ & $30(7)$ & $29(6)$ & 0.3 \\
\hline \multicolumn{4}{|l|}{ Comorbidities, $n(\%)$} \\
\hline Coronary artery disease & $85(18)$ & $17(21)$ & 0.57 \\
\hline Heart failure & $13(3)$ & $5(6)$ & 0.12 \\
\hline Cerebrovascular disease & $25(5)$ & $6(7)$ & 0.47 \\
\hline Peripheral artery disease & $17(4)$ & $2(3)$ & 0.59 \\
\hline Diabetes mellitus & $93(20)$ & $19(24)$ & 0.48 \\
\hline Hypertension & $288(62)$ & $55(68)$ & 0.32 \\
\hline Atrial fibrillation & $17(4)$ & $8(10)$ & 0.01 \\
\hline Recent smoker* & $74(16)$ & $20(25)$ & 0.06 \\
\hline Obstructive lung disease $\dagger$ & $68(15)$ & $9(11)$ & 0.40 \\
\hline Arthritis & $150(32)$ & $21(26)$ & 0.25 \\
\hline 6MWT distance in metres, mean (SD) & $477(100)$ & $457(97)$ & 0.10 \\
\hline \multicolumn{4}{|l|}{ Renal function, $(n=515), n(\%) \ddagger$} \\
\hline $\mathrm{eGFR} \geq 60 \mathrm{~mL} \cdot \mathrm{min}^{-1}(1.73 \mathrm{~m})^{-2}$ & $393(90)$ & $62(79)$ & 0.03 \\
\hline eGFR 30 to $59 \mathrm{~mL} \cdot \mathrm{min}^{-1}(1.73 \mathrm{~m})^{-2}$ & $40(9)$ & $14(18)$ & \\
\hline $\mathrm{eGFR}<30 \mathrm{~mL} \cdot \mathrm{min}^{-1}(1.73 \mathrm{~m})^{-2}$ or dialysis & $4(1)$ & $2(3)$ & \\
\hline \multicolumn{4}{|l|}{ Natriuretic peptide concentration $(n=515), n(\%)$} \\
\hline NT pro-BNP $<100 \mathrm{ng} \cdot \mathrm{L}^{-1}$ & $241(55)$ & $29(37)$ & 0.003 \\
\hline NT pro-BNP $100 \mathrm{ng} \cdot \mathrm{L}^{-1}$ to $<300 \mathrm{ng} \cdot \mathrm{L}^{-1}$ & $135(31)$ & $28(36)$ & \\
\hline NT pro-BNP ng. $\mathrm{L}^{-1} \geq 300 \mathrm{ng} \cdot \mathrm{L}^{-1}$ & $61(14)$ & $21(27)$ & \\
\hline \multicolumn{4}{|l|}{ ASA Physical Status Score, $n(\%)$} \\
\hline I & $19(4)$ & $3(4)$ & 0.48 \\
\hline II & $267(58)$ & $40(49)$ & \\
\hline III & $172(37)$ & $36(44)$ & \\
\hline IV & $6(1)$ & $2(1)$ & \\
\hline \multicolumn{4}{|l|}{ Revised Cardiac Risk Index $(n=518), n(\%)$} \\
\hline Class 1 & $156(34)$ & $11(14)$ & 0.001 \\
\hline Class 2 & $191(41)$ & $41(51)$ & \\
\hline Class 3 & $76(16)$ & $19(24)$ & \\
\hline Class 4 & $17(4)$ & $7(9)$ & \\
\hline \multicolumn{4}{|l|}{ Operative characteristics } \\
\hline \multicolumn{4}{|l|}{ Procedure type, $n(\%)$} \\
\hline Vascular & $14(3)$ & $3(4)$ & 0.005 \\
\hline Intrathoracic & $21(5)$ & $1(1)$ & \\
\hline Intraperitoneal or retroperitoneal & $136(29)$ & $43(53)$ & \\
\hline Urologic or gynecologic & $141(30)$ & $16(20)$ & \\
\hline Head-and-neck & $51(11)$ & $6(7)$ & \\
\hline Orthopedic & 79 (17) & $9(11)$ & \\
\hline Other & $22(5)$ & $3(4)$ & \\
\hline
\end{tabular}


Table 2 continued

\begin{tabular}{llll}
\hline Variable & $\begin{array}{l}\text { Mild or no } \\
\text { complications }(n=464)\end{array}$ & $\begin{array}{l}\text { Moderate or severe } \\
\text { complications }(n=81)\end{array}$ \\
\hline $\begin{array}{l}\text { Postoperative hospitalization } \\
\text { Postoperative length-of-stay (day), median [IQR] }\end{array}$ & $3[2-5]$ & $10[7.5-16.5]$ & $<0.001$ \\
\hline
\end{tabular}

Categorical variables were compared using $\chi^{2}$ or Fisher's exact test, while continuous variables were compared using the two-sample $t$ test or Mann-Whitney U test

ASA = American Society of Anesthesiologists; BMI = body mass index; eGFR = estimated glomerular filtration rate; IQR = interquartile range; NT pro-BNP = N-terminal pro-B-type natriuretic peptide; $6 \mathrm{MWT}=$ six-minute walk test; $\mathrm{SD}=$ standard deviation

*Current smoker or quit within the previous year

${ }^{\dagger}$ Prior diagnosis of asthma, reactive airways disease, chronic obstructive lung disease, chronic bronchitis, or emphysema

* Estimated glomerular filtration rate was calculated using the preoperative serum creatinine concentration and Chronic Kidney Disease Epidemiology Collaboration equation

- Revised Cardiac Risk Index scores were calculated using a modified definition of diabetes mellitus (i.e., any prior diagnosis of diabetes mellitus, as opposed to requirement for insulin therapy)

Cardiopulmonary exercise testing subset analysis

Ninety-two percent $(n=501)$ of the primary analysis cohort attempted preoperative CPET. The test was terminated early in 67 patients, with the most common reason being an inability to pedal ( $n=23)$ (eTable 4, ESM). There was a modest correlation between either 6MWT distance and peak oxygen consumption $(\rho=0.390 ; P<$ $0.001)$ or 6 MWT distance and anaerobic threshold $(\rho=$ $0.311 ; P<0.001$ ) (Fig. 2).

\section{Sensitivity analyses}

The adjusted association of the 6MWT distance with the primary and secondary outcomes was qualitatively unchanged in sensitivity analyses with bootstrap internal validation and different model covariates (eTable 4, ESM).

\section{Post hoc analyses}

Two hundred and seventy-three participants (50\%) performed CPET and the 6MWT on the same day. The mean (SD) 6MWT distance did not significantly differ between participants who performed CPET and the 6MWT on the same day compared with those who did not [480 (99) $\mathrm{m} v s 467$ (101) m, respectively; mean difference, -13 $\mathrm{m} ; 95 \% \mathrm{CI},-29$ to $4 ; P=0.14]$. The association of preoperative 6MWT with moderate or severe complications was modified $(P=0.045)$ by performance of CPET and the 6MWT on the same day (model presented in eTable 5, ESM). Reduced preoperative 6MWT was associated with increased odds of moderate or severe complications (aOR, 1.79 per $100 \mathrm{~m}$ decrease; 95\% CI, 1.20 to 2.66) among individuals who did not perform CPET on the same day as the 6MWT, but not among
Table 3 Adjusted association of 6MWT distance and other clinical risk factors with moderate or severe postoperative in-hospital complications

\begin{tabular}{|c|c|c|}
\hline Risk factor & $\begin{array}{l}\text { Odds ratio }(95 \% \mathrm{CI}) \text { for } \\
\text { moderate or severe } \\
\text { complications }\end{array}$ & $P$ value \\
\hline \multicolumn{3}{|l|}{ 6MWT distance } \\
\hline Per 100 -m decrease & $1.32(1.01$ to 1.73$)$ & 0.045 \\
\hline \multicolumn{3}{|l|}{ Surgical procedure } \\
\hline Moderate-risk procedure & Reference & \\
\hline High-risk procedure & $3.43(2.04$ to 5.78$)$ & $<0.001$ \\
\hline \multicolumn{3}{|l|}{ Sex } \\
\hline Male & Reference & \\
\hline Female & $0.62(0.36$ to 1.07$)$ & 0.09 \\
\hline \multicolumn{3}{|l|}{ Age } \\
\hline Per $10-y r$ increase & $1.11(0.87$ to 1.42$)$ & 0.42 \\
\hline \multicolumn{3}{|c|}{$\begin{array}{l}\text { Model was fit using } 545 \text { observations with complete data. The } \\
\text { multivariable regression model had a c-index of } 0.68 \text { and Hosmer- } \\
\text { Lemeshow goodness-of-fit statistic } P \text { value of } 0.84\end{array}$} \\
\hline \multicolumn{3}{|c|}{$\mathrm{CI}=$ confidence interval $; 6 \mathrm{MWT}=$ six-minute walk test } \\
\hline
\end{tabular}

individuals who did (aOR, 1.06 per $100 \mathrm{~m}$ decrease; $95 \%$ CI, 0.75 to 1.50 ).

\section{Discussion}

In this secondary analysis of a multicentre prospective cohort study, reduced preoperative 6MWT distance was associated with increased odds of moderate or severe inhospital complications, even after adjusting for other easily measured clinical characteristics such as demographics and surgery type. Nonetheless, the strength of statistical evidence for this association was modest; furthermore, 
Table 4 Adjusted association of 6MWT distance and other clinical risk factors with 30-day death or myocardial injury

\begin{tabular}{lll}
\hline Risk factor & $\begin{array}{l}\text { Odds ratio }(95 \% \mathrm{CI}) \text { for 30-day death } \\
\text { or myocardial injury }\end{array}$ & $P$ value \\
\hline 6MWT distance* & & \\
$500 \mathrm{~m}$ vs $600 \mathrm{~m}$ & $1.00(0.65$ to 2.90$)$ & 0.49 \\
$400 \mathrm{~m}$ vs $600 \mathrm{~m}$ & $1.61(0.72$ to 3.56$)$ & \\
$300 \mathrm{~m}$ vs $600 \mathrm{~m}$ & $1.13(0.46$ to 2.79$)$ & \\
Sex & & \\
Male & Reference & \\
Female & $1.03(0.56$ to 1.90$)$ & \\
Age & & \\
Per 10-yr & $1.81(1.32$ to 2.50$)$ & \\
increase & & \\
Revised Cardiac & & \\
Risk Index & & \\
Class 1 & Reference & \\
Class 2 & $1.58(0.73$ to 3.40$)$ & \\
Class 3 & $3.15(1.35$ to 7.36$)$ & \\
Class 4 & $3.33(1.00$ to 11.08$)$ & \\
NT pro-BNP & & \\
concentration & & \\
$<300 \mathrm{ng} \cdot \mathrm{L}^{-1}$ & Reference \\
$\geq 300 \mathrm{ng} \cdot \mathrm{L}^{-1}$ & $1.48(0.75$ to 2.91$)$ & \\
\hline
\end{tabular}

Model was fit using 515 observations with complete data. The multivariable regression model had a c-index of 0.73 and HosmerLemeshow goodness-of-fit statistic $P$ value of 0.31

$\mathrm{CI}=$ confidence interval; $6 \mathrm{MWT}=$ six-minute walk test

*Adjusted odds ratios were extrapolated based on a restricted cubic spline function with four knots (to account for non-linear association of 6MWT distance with the outcome)

we did not find evidence that 6MWT distance provided incremental prognostic information for predicting postoperative complications. By comparison, 6MWT distance had no meaningful association with or myocardial injury or 30-day postoperative death. These overall findings were consistent after accounting for other preoperative risk factors and internal validation using bootstrap resampling.

While previous studies, including the primary report of the METS study, have shown that metrics derived from CPET performance can predict postoperative complications, ${ }^{1,3}$ there are important practical limitations to the widespread implementation of preoperative CPET. Stated simply, it requires capital, technical, and professional costs that render it infeasible in many settings. Within that context, the 6MWT has several attractive features, including relatively low resource requirements, standardized protocols, and excellent tolerability. For example, in the cohort included in this secondary analysis, only about $1 \%$ of $6 \mathrm{MWT}$ sessions were terminated early compared with $13 \%$ of CPET sessions.

Our study also confirmed the validity of the 6MWT as a measure of preoperative cardiopulmonary fitness based on the correlation of 6MWT distance with peak oxygen consumption and anaerobic threshold. Nevertheless, the magnitude of this correlation was weaker than was observed in other cohort studies where the correlations were instead in the range of 0.64 to $0.75 .^{7,29,30}$ Nonetheless, the 6MWT and CPET had similar profiles with respect to preoperative risk assessment, in that both 6MWT and peak oxygen consumption were predictive of moderate or severe complications but not myocardial injury. ${ }^{1}$ Notably, CPET measurements were determined by investigators across 15 participating hospitals, which raises the possibility of observer and measurement bias between centres. It is possible that central interpretation of CPET data could have altered the observed correlation between 6MWT distance and CPET performance.

The results are an important addition to the literature on the validity of the preoperative 6MWT in estimating perioperative risk outside the specialized context of lung cancer resection surgery. Some prior studies have found associations between 6MWT distance and outcomes, ${ }^{10-15}$ while others have not. ${ }^{16,17}$ These studies typically had small sample sizes with fewer than 150 participants, and included specific subsets of surgical patients, thereby limiting the generalizability of their results. In addition, there was considerable variability in how these studies defined complications. Some studies used standardized measures such as the Clavien-Dindo classification scheme, ${ }^{11,15}$ while others used self-defined cardiopulmonary complications ${ }^{13}$ or solely pulmonary complications. ${ }^{12,14,17}$ Compared with this prior work, our present study included a moderately large cohort of patients having major non-cardiac surgery and systematically followed up participants to ascertain several clinically and prognostically important outcomes.

Prior studies have focused on identifying thresholds of 6MWT performance to identify patients at elevated risk of postoperative complications, especially since simple thresholds may be attractive to clinicians. ${ }^{12,14,15}$ Nonetheless, our results would caution against interpreting preoperative 6MWT performance based on this approach. From a methodological perspective, dichotomization of continuous predictor variables in clinical prediction models can lead to loss of statistical power, residual confounding, and serious bias. ${ }^{31,32}$ Thus, allocation of patients into one of two risk categories based on preoperative 6MWT performance can lead to overly simple and incorrect clinical decisions. Instead, there appears to be a linear association between 6MWT distance and complications, whereby risk plausibly 

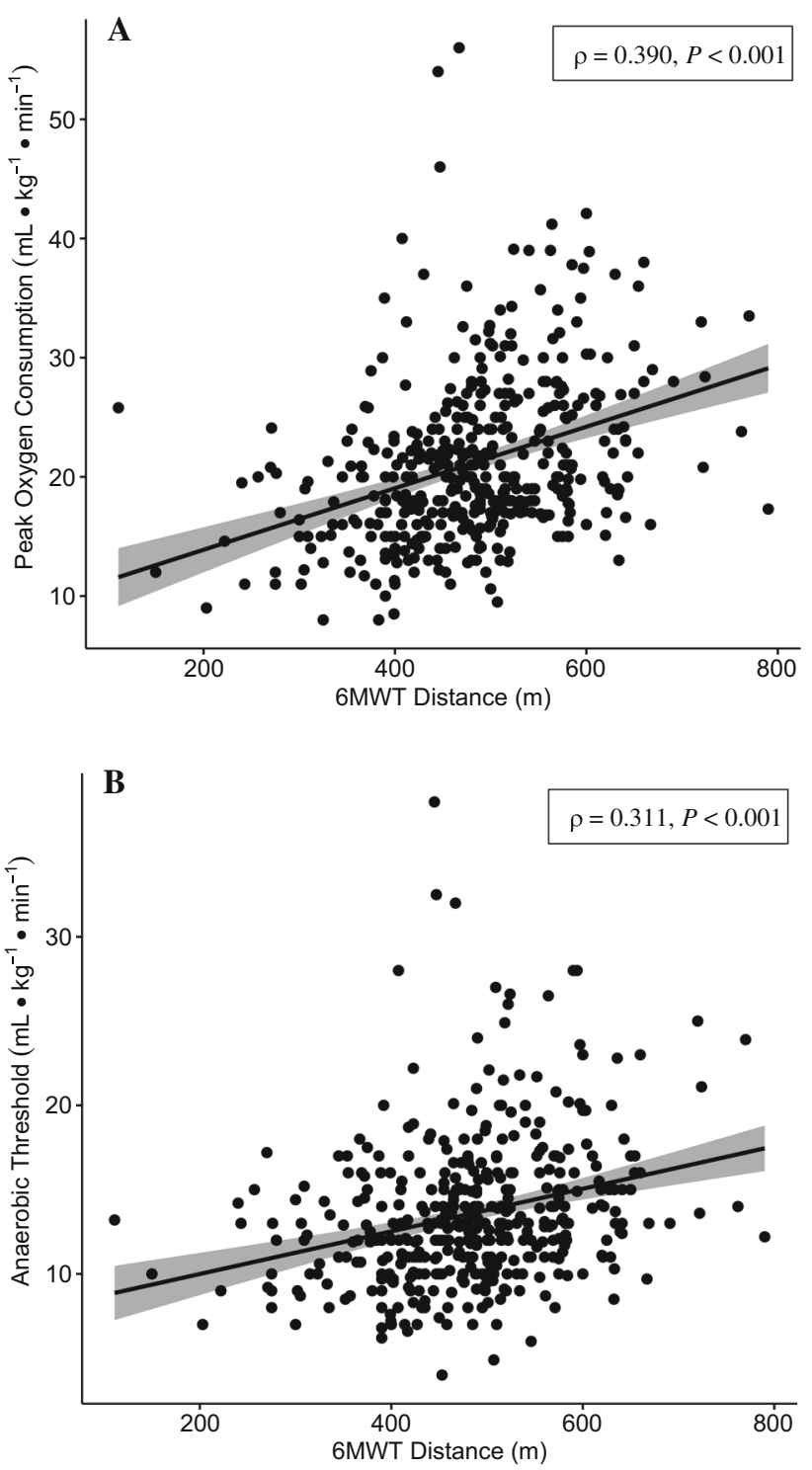

Fig. 2 Correlation between the six-minute walk test (6MWT) distance and peak oxygen consumption or $\mathrm{VO}_{2}$ peak (panel $\mathrm{A}$ ) and anaerobic threshold or AT (panel B)

increased on a continuum as 6MWT performance decreased. We found that each $100 \mathrm{~m}$ decrease in 6MWT distance was associated with an approximately $30 \%$ increase in the adjusted odds of complications. Thus, clinical prediction rules that include actual 6MWT distance may be more appropriate for risk-stratifying patients.

Our study has multiple strengths, including the prospective, international, multicentre, and diverse study cohort. There was minimal patient loss to follow-up and minimal incomplete data. The primary outcome, moderate or severe complications as defined by the modified Clavien-Dindo classification system, is an accepted measure of postoperative morbidity. In addition, the secondary outcome, 30-day death or myocardial injury, is an objective and prognostically important endpoint.
Our study has several important limitations that should be considered when interpreting the findings. First, 6MWT performance can be affected by factors other than cardiopulmonary fitness, such as patient comorbidities (e.g., peripheral artery disease, arthritis), coordination, and frailty. Second, the requirement that participants perform two preoperative exercise tests (6MWT and CPET) before surgery likely introduced selection bias, whereby healthier patients were more likely to participate in this study. Consistent with this possibility, the risk of 30-day death or myocardial infarction in the METS-6MWT substudy cohort was only one-third of that seen in more generalizable cohorts where intensive preoperative exercise testing was not required. ${ }^{18,33}$ Thus, it is possible the substudy cohort had skewed distributions of both 6MWT results and other predictors of postoperative complications that affected the strength of association between 6MWT distance and outcomes. Third, the study design allowed for preoperative CPET and 6MWT to be performed on the same day, largely for pragmatic reasons. While such participants had to rest for at least two hours between the exercise tests, exploratory subgroup analyses suggest that preoperative 6MWT distance was predictive of moderate or severe complications only if the 6MWT and CPET were performed on separate days. From a clinical perspective, these findings suggest that participants who had performed CPET and the 6MWT on the same day were not sufficiently rested between the two exercise tests. Since subgroup analyses should be interpreted very cautiously, ${ }^{34}$ our findings warrant replication in a future study that specifically disallows any other exercise testing on the same day as the preoperative 6MWT. Fourth, while the sample size of this study was larger than previous studies, it is likely still not sufficiently large for robust development of a clinical prediction model. Even a relatively simple multivariable regression model with the same four predictor variables used in our primary analysis (age, sex, surgical procedure, 6MWT distance) should likely include 12 parameters to allow for flexible modelling of continuous variables (age, 6MWT distance), ${ }^{35}$ and more clinically appropriate categorization of surgical procedures. ${ }^{36}$ Assuming the same primary outcome rate (15\%) observed in the 6MWT-METS substudy, a minimum sample size of 697 is needed to develop a plausible clinical prediction model. ${ }^{37}$

\section{Conclusions}

In this secondary analysis of a multicentre prospective cohort study, preoperative 6MWT distance had a modest ability to predict moderate or severe complications, but not 30-day death or myocardial injury, after major inpatient 
non-cardiac surgery. Further large, prospective studies are needed to determine the optimal role of the 6MWT as a simple objective exercise test to inform preoperative risk stratification for non-cardiac surgery.

Author contributions All authors contributed to the study conception and design. Brian H. Cuthbertson, Mark A. Shulman, Paul S. Myles, and Duminda N. Wijeysundera contributed to the acquisition of data. Ryan J. Ramos and Duminda N. Wijeysundera contributed to the analysis of data. All authors contributed to the interpretation of data. Ryan J. Ramos wrote the first draft of the manuscript, and all authors revised the manuscript for important intellectual content.

6MWT-METS Substudy Investigators Study Site Investigators: Australia-Alfred Hospital: P S Myles (Site Co-Lead), M A Shulman (Site Co-Lead), S Wallace, C Farrington, B Thompson (Site CPET Lead), M Ellis, B Borg; John Hunter Hospital: R K Kerridge (Site Lead), J Douglas, J Brannan, J Pretto; Nambour General Hospital: M G Godsall (Site Co-Lead), N Beauchamp (Site Co-Lead), S Allen, A Kennedy, E Wright, J Malherbe; Peter McCallum Cancer Centre: H Ismail (Site Co-Lead), B Riedel (Site Co-Lead), A Melville, H Sivakumar, A Murmane, $\mathrm{K}$ Kenchington, Y Kirabiyik; Prince Charles Hospital: U Gurunathan (Site Lead), C Stonell, K Brunello, K Steele, O Tronstad, P Masel, A Dent, E Smith, A Bodger, M Abolfathi; Princess Alexandra Hospital: P Sivalingam (Site CoLead), A Hall (Site Co-Lead); Royal Adelaide Hospital: T W Painter (Site Co-Lead), S Macklin (Site Co-Lead), A Elliott, A M Carrera; Royal Hobart Hospital: N C S Terblanche (Site Lead); S Pitt, J Samuels, C Wilde; Royal Melbourne Hospital: K Leslie (Site Lead), A MacCormick; Western Health: D Bramley (Site Lead), A M Southcott, J Grant, H Taylor, S Bates, M Towns, A Tippett, F Marshall. Canada-Sunnybrook Health Sciences Centre: C J L McCartney (Site Co-Lead), S Choi (Site Co-Lead), P Somascanthan, K Flores; Toronto General Hospital: D N Wijeysundera (Site Co-Lead), W S Beattie (Site Co-Lead), K Karkouti, H A Clarke, A Jerath, S A McCluskey, M Wasowicz, J T Granton (Site CPET Lead), L Day, J Pazmino-Canizares; Toronto Rehabilitation Institute-Rumsey Centre: $\mathrm{P}$ Oh (Site Lead), R Belliard, L Lee, K Dobson; Toronto Western Hospital: M Stanbrook (Site CPET Lead). New Zealand - Auckland City Hospital: K Hagen (Site Lead), D Campbell, T Short, J Van Der Westhuizen, K Higgie, H Lindsay, R Jang, C Wong, D Mcallister, M Ali, J Kumar, E Waymouth, C Kim; Middlemore Hospital: J Dimech (Site Co-Lead), M Lorimer (Site Co-Lead), J Tai, R Miller, R Sara, A Collingwood, S Olliff, S Gabriel, H Houston; Wellington Hospital: P Dalley (Site Lead), S Hurford, A Hunt, L Andrews, L Navarra, A Jason-Smith, H Thompson, N McMillan, G Back. International and National Coordinators-B.H. Cuthbertson (International Co-Principal Investigator), D.N. Wijeysundera (International Co-Principal Investigator), R.M. Pearse, P.S. Myles, T.E.F. Abbott, M.A. Shulman. Central Project Office Operations Committee-B.H. Cuthbertson, D.N. Wijeysundera, E. Torres, A. Ambosta, M. Melo,
M. Mamdani, K.E. Thorpe, R.M. Pearse, T.E. F. Abbott, P.S. Myles, M.A. Shulman, S. Wallace, C. Farrington, B.L. Croal. CPET Methods Committee-M.P.W.Grocott, J.T. Granton, P. Oh, B. Thompson, D. Levett. Outcome Adjudication Committee-G. Hillis (Chair), W.S. Beattie, H.C. Wijeysundera. International Steering Committee-B.H. Cuthbertson (International Co-Principal Investigator), D.N. Wijeysundera (International Co-Principal Investigator), R.M. Pearse, M.A. Shulman, T.E.F. Abbott, E. Torres, A. Ambosta, B.L. Croal, J.T. Granton, K.E. Thorpe, M.P.W. Grocott, C. Farrington, S. Wallace, P.S. Myles.

Disclosures None.

Funding statement Dr. Wijeysundera, Dr. Ladha, and Dr. Cuthbertson are supported in part by Merit Awards from the Department of Anesthesiology and Pain Medicine at the University of Toronto. Dr. Ladha is supported in part by an International Anesthesia Research Society (IARS) Mentored Research Award. Dr. Wijeysundera is supported in part by the Endowed Chair in Translational Anesthesiology Research at St. Michael's Hospital and University of Toronto. Dr. Myles is supported by an Australian National Health and Medical Research Council Practitioner Fellowship. The METS study was supported by grants from the Canadian Institutes of Health Research; Heart and Stroke Foundation of Canada; Ontario Ministry of Health and Long-Term Care; Ontario Ministry of Research, Innovation and Science; United Kingdom (UK) National Institute of Academic Anaesthesia; UK Clinical Research Collaboration; Australian and New Zealand College of Anaesthetists; and Monash University (Melbourne, Victoria, Australia). These sponsors had no role in the design and conduct of the METS study; collection, management, analysis, and interpretation of the data; preparation, review, or approval of this paper; and decision to submit this manuscript for publication.

Editorial responsibility This submission was handled by Dr. Hilary P. Grocott, Editor-in-Chief, Canadian Journal of Anesthesia.

Open Access This article is licensed under a Creative Commons Attribution-NonCommercial 4.0 International License, which permits any non-commercial use, sharing, adaptation, distribution and reproduction in any medium or format, as long as you give appropriate credit to the original author(s) and the source, provide a link to the Creative Commons licence, and indicate if changes were made. The images or other third party material in this article are included in the article's Creative Commons licence, unless indicated otherwise in a credit line to the material. If material is not included in the article's Creative Commons licence and your intended use is not permitted by statutory regulation or exceeds the permitted use, you will need to obtain permission directly from the copyright holder. To view a copy of this licence, visit http://creativecommons.org/licenses/ by-nc/4.0/. 


\section{Appendix 1}

\section{See Table 5.}

Table 5 Inclusion criteria for the METS study (one or more had to be present)

\begin{tabular}{|c|c|}
\hline Risk factor & Definition \\
\hline $\begin{array}{l}\text { Intermediate to high-risk } \\
\text { surgery }\end{array}$ & Intraperitoneal, intrathoracic, or major vascular (supra-inguinal or lower extremity vascular) procedures \\
\hline Coronary artery disease & $\begin{array}{l}\text { History of angina; myocardial infarction; positive exercise, nuclear or echocardiographic stress test; resting wall } \\
\text { motion abnormalities on echocardiogram; coronary angiography with evidence of } \geq 50 \% \text { vessel stenosis; or } \\
\text { electrocardiogram with pathologic Q-waves in two contiguous leads }\end{array}$ \\
\hline Heart failure & History of heart failure or diagnostic chest $x$-ray (i.e., pulmonary vascular redistribution or pulmonary edema) \\
\hline Cerebrovascular disease & History of stroke or transient ischemic attack; or imaging (CT or MRI) evidence of previous stroke \\
\hline Diabetes mellitus & Requirement for insulin or oral hypoglycemic therapy \\
\hline $\begin{array}{l}\text { Preoperative renal } \\
\text { insufficiency }\end{array}$ & $\begin{array}{l}\text { Requirement for renal replacement therapy before surgery, or estimated glomerular filtration rate } \leq 60 \\
\mathrm{~mL} \cdot \mathrm{min}^{-1} 1.73 \mathrm{~m}^{-2}\end{array}$ \\
\hline Peripheral arterial disease & $\begin{array}{l}\text { History of peripheral arterial disease; ischemic intermittent claudication; rest pain; lower limb revascularization } \\
\text { procedure; peripheral arterial obstruction of } \geq 50 \% \text { luminal diameter; or resting ankle/arm systolic blood pressure } \\
\text { ratio } \leq 0.90\end{array}$ \\
\hline Hypertension & Doctor's diagnosis of hypertension \\
\hline Smoker & History of smoking within 1 year before surgery \\
\hline Advanced age & $\geq 70 \mathrm{yr}$ \\
\hline
\end{tabular}

$\mathrm{CT}=$ computed tomography; $\mathrm{MRI}=$ magnetic resonance imaging

\section{Appendix 2}

See Table 6.

Table 6 Exclusion criteria

At the time of approach for recruitment to study, inadequate time to feasible complete CPET before surgery (defined as $<24$ hr)

Planned use of CPET or 6MWT for preoperative risk stratification independent of METS study protocol

Planned surgery exclusively performed by an endovascular approach (e.g., endovascular aortic aneurysm repair)

Presence of an automated implantable cardioverter-defibrillator

Known or suspected pregnancy

Previous enrolment in the METS study

Active cardiac conditions, ${ }^{38}$ absolute contraindications to CPET (American Thoracic Society and American College of Chest Physicians guidelines), ${ }^{39}$ contraindications to $6 \mathrm{MWT},{ }^{20}$ and conditions expected to preclude CPET or 6MWT (e.g., lower limb amputation, severe claudication, severe orthopedic impairment)

Systolic blood pressure $\geq 180 \mathrm{mmHg}$ and diastolic blood pressure $\geq 100 \mathrm{mmHg}$ at the time of study recruitment

$\mathrm{CPET}=$ cardiopulmonary exercise testing; METS $=$ Measurement of Exercise Tolerance before Surgery; $6 \mathrm{MWT}=$ six-minute walk test 


\section{Appendix 3}

See Table 7.

Table 7 Definitions of in-hospital postoperative complications

\begin{tabular}{|c|c|}
\hline Complication & Definition \\
\hline Non-fatal cardiac arrest & $\begin{array}{l}\text { Successful resuscitation from documented (or presumed) ventricular fibrillation, sustained ventricular } \\
\text { tachycardia, asystole, or pulseless electrical activity }\end{array}$ \\
\hline Heart failure & $\begin{array}{l}\text { Presence of both } \\
\text { - clinical findings (i.e., elevated jugular venous pressure, respiratory rates, crepitations, } S_{3} \text { heart sounds) } \\
\text { - radiological findings (i.e., vascular redistribution, interstitial or frank pulmonary edema) }\end{array}$ \\
\hline Stroke & New focal neurologic deficit, suspected to be vascular in origin, with signs/symptoms lasting $\geq 24 \mathrm{hr}$ \\
\hline Transient ischemic attack & Transient focal neurologic deficit that lasts less than $24 \mathrm{hr}$ and is thought to be vascular in origin \\
\hline Respiratory failure & $\begin{array}{l}\text { Need for tracheal intubation and mechanical ventilation after patient has completed surgery, been successfully } \\
\text { extubated, and breathing spontaneously for }>1 \mathrm{hr}\end{array}$ \\
\hline Pneumonia & $\begin{array}{l}\text { Documented hypoxemia }\left(\mathrm{PaO}_{2} / \mathrm{F}_{1} \mathrm{O}_{2} \text { ratio } \leq 250 \mathrm{mmHg} \text { ) or fever (temperature }>37.5^{\circ} \mathrm{C} \text { ) with either: }\right. \\
\text { 1. Rales or dullness to percussion on chest examination and any of (i) new onset of purulent sputum or change } \\
\text { in sputum character; (ii) organism isolated from blood culture; or (iii) pathogen isolated from trans-tracheal } \\
\text { aspirate, bronchial brushing, or biopsy } \\
\text { 2. New or progressive infiltrate, consolidation, cavitation, or pleural effusion on chest radiograph and any of } \\
\text { (a) criteria i, ii, or iii above; (b) detection of virus or viral antigen in respiratory secretions; (c) diagnostic } \\
\text { antibody titers; or (d) histopathologic evidence of pneumonia }\end{array}$ \\
\hline Surgical site infection & $\begin{array}{l}\text { Physician diagnosis of surgical site infection during: } \\
\text { - index hospitalization } \\
\text { - outpatient visit, hospital re-admission, or emergency room visit within } 30 \text { days after index surgery }\end{array}$ \\
\hline Deep venous thrombosis & $\begin{array}{l}\text { Any of the following during index hospitalization: } \\
\text { 1. Persistent intraluminal filling defect on contrast venography } \\
\text { 2. One or more non-compressible venous segments on B mode compression ultrasonography } \\
\text { 3. Clearly defined intraluminal filling defect on contrast enhanced computed tomography }\end{array}$ \\
\hline Pulmonary embolism & $\begin{array}{l}\text { Any of the following during index hospitalization: } \\
\text { 1. High probability ventilation/perfusion lung scan } \\
\text { 2. Intraluminal filling defect of segmental or larger artery on a helical CT scan } \\
\text { 3. Intraluminal filling defect on pulmonary angiography } \\
\text { 4. A positive diagnostic test for deep venous thrombosis (e.g., positive compression ultrasound) plus low or } \\
\text { intermediate probability ventilation/perfusion lung scan, or non-diagnostic (sub-segmental defects or } \\
\text { technically inadequate study) helical CT scan }\end{array}$ \\
\hline Significant bleeding & $\begin{array}{l}\text { Blood loss with any of the following characteristics: } \\
\text { 1. Results in drop in hemoglobin concentration of } 30 \mathrm{~g} \cdot \mathrm{L}^{-1} \text { or more } \\
\text { 2. Leads to red cell transfusion or re-operation } \\
\text { 3. Considered the cause of death }\end{array}$ \\
\hline $\begin{array}{l}\text { Unexpected admission to critical } \\
\text { care unit }\end{array}$ & Unexpected admission to critical care unit, intensive care unit, step-down unit, or high-dependency unit \\
\hline Re-operation & Return to operating room within index hospitalization \\
\hline $\begin{array}{l}\text { Severity of postoperative } \\
\text { complications }\end{array}$ & $\begin{array}{l}\text { 1. Mild: only temporary harm that did not require clinical treatment } \\
\text { 2. Moderate: required clinical treatment but without significantly prolonged hospital stay. Did not usually } \\
\text { result in permanent harm and where this did occur, there was no associated functional limitation } \\
\text { 3. Severe: required clinical treatment and resulted in significant prolongation of hospital stay and/or permanent } \\
\text { functional limitation } \\
\text { 4. Fatal: death from the complication }\end{array}$ \\
\hline
\end{tabular}

$\mathrm{CT}=$ computerized tomography; ECG = electrocardiogram; $\mathrm{PaO}_{2} / \mathrm{F}_{1} \mathrm{O}_{2}=$ arterial oxygen partial pressure/fraction of inspired oxygen 


\section{References}

1. Wijeysundera DN, Pearse RM, Shulman MA, et al. Assessment of functional capacity before major non-cardiac surgery: an international, prospective cohort study. Lancet 2018; 391: 2631-40.

2. Guazzi M, Adams V, Conraads V, et al. EACPR/AHA Scientific Statement. Clinical recommendations for cardiopulmonary exercise testing data assessment in specific patient populations. Circulation 2012; 126: 2261-74.

3. Moran J, Wilson F, Guinan E, McCormick P, Hussey J, Moriarty $J$. Role of cardiopulmonary exercise testing as a risk-assessment method in patients undergoing intra-abdominal surgery: a systematic review. Br J Anaesth 2016; 116: 177-91.

4. Lai $C W$, Minto $G$, Challand $C P$, et al. Patients' inability to perform a preoperative cardiopulmonary exercise test or demonstrate an anaerobic threshold is associated with inferior outcomes after major colorectal surgery. Br J Anaesth 2013; 111: 607-11.

5. Holland AE, Spruit MA, Troosters T, et al. An official European Respiratory Society/American Thoracic Society technical standard: field walking tests in chronic respiratory disease. Eur Respir J 2014; 44: 1428-46.

6. Singh SJ, Puhan MA, Andrianopoulos V, et al. An official systematic review of the European Respiratory Society/American Thoracic Society: measurement properties of field walking tests in chronic respiratory disease. Eur Respir J 2014; 44: 1447-78.

7. Sinclair RC, Batterham AM, Davies S, Cawthorn L, Danjoux GR. Validity of the 6 min walk test in prediction of the anaerobic threshold before major non-cardiac surgery. Br J Anaesth 2012; 108: $30-5$.

8. Marjanski T, Wnuk D, Bosakowski D, Szmuda T, Sawicka W, Rzyman W. Patients who do not reach a distance of $500 \mathrm{~m}$ during the 6-min walk test have an increased risk of postoperative complications and prolonged hospital stay after lobectomy. Eur $\mathbf{J}$ Cardiothorac Surg 2015; 47: e213-9.

9. Wesolowski S, Orlowski TM, Kram M. The 6-min walk test in the functional evaluation of patients with lung cancer qualified for lobectomy. Interact Cardiovasc Thorac Surg 2020; 30: 559-64.

10. Awdeh H, Kassak K, Sfeir P, Hatoum H, Bitar H, Husari A. The SF-36 and 6-minute walk test are significant predictors of complications after major surgery. World J Surg 2015; 39: 1406-12.

11. Lee L, Schwartzman K, Carli F, et al. The association of the distance walked in 6 min with pre-operative peak oxygen consumption and complications 1 month after colorectal resection. Anaesthesia 2013; 68: 811-6.

12. Miccichè $V$, Esposito $C$, Santaniello $W$, et al. Six-minute walk test in pre-operative evaluation of patients for upper abdominal surgery. Eur J Anaesthesiol 2019; 36: 164-6.

13. Arruda KA, Cataneo DC, Cataneo AJ. Surgical risk tests related to cardiopulmonary postoperative complications: comparison between upper abdominal and thoracic surgery. Acta Cir Bras 2013; 28: 458-66.

14. Keeratichananont $W$, Thanadetsuntorn $C$, Keeratichananont $S$. Value of preoperative 6-minute walk test for predicting postoperative pulmonary complications. Ther Adv Respir Dis 2016; 10: 18-25.

15. Inoue $T$, Ito $S$, Kanda $M$, et al. Preoperative six-minute walk distance as a predictor of postoperative complication in patients with esophageal cancer. Dis Esophagus 2020; DOI: https://doi. org/10.1093/dote/doz050.

16. Moriello $C$, Mayo NE, Feldman L, Carli F. Validating the sixminute walk test as a measure of recovery after elective colon resection surgery. Arch Phys Med Rehabil 2008; 89: 1083-9.
17. Paisani DM, Fiore JF Jr, Lunardi AC, et al. Preoperative 6-min walking distance does not predict pulmonary complications in upper abdominal surgery. Respirology 2012; 17: 1013-7.

18. Shulman MA, Cuthbertson BH, Wijeysundera DN, et al. Using the 6-minute walk test to predict disability-free survival after major surgery. Br J Anaesth 2019; 122: 111-9.

19. Wijeysundera DN, Pearse RM, Shulman MA, et al. Measurement of Exercise Tolerance before Surgery (METS) study: a protocol for an international multicentre prospective cohort study of cardiopulmonary exercise testing prior to major non-cardiac surgery. BMJ Open 2016; DOI: https://doi.org/10.1136/bmjopen2015-010359.

20. ATS Committee on Proficiency Standards for Clinical Pulmonary Function Laboratories. ATS statement: guidelines for the sixminute walk test. Am J Respir Crit Care Med 2002; 166: 111-7.

21. Dindo D, Demartines N, Clavien PA. Classification of surgical complications: a new proposal with evaluation in a cohort of 6336 patients and results of a survey. Ann Surg 2004; 240: 205-13.

22. International, Surgical Outcomes Study (ISOS) group. Prospective observational cohort study on grading the severity of postoperative complications in global surgery research. Br J Surg 2019; 106: e73-80.

23. Thygesen K, Alpert JS, Jaffe AS, et al. Fourth universal definition of myocardial infarction (2018). Eur Heart J 2019; 40: 237-69.

24. Lee TH, Marcantonio ER, Mangione CM, et al. Derivation and prospective validation of a simple index for prediction of cardiac risk of major noncardiac surgery. Circulation 1999; 100: 1043-9.

25. Duceppe E, Parlow J, MacDonald $P$, et al. Canadian Cardiovascular Society guidelines on perioperative cardiac risk assessment and management for patients who undergo noncardiac surgery. Can J Cardiol 2017; 33: 17-32.

26. DeLong ER, DeLong DM, Clarke-Pearson DL. Comparing the areas under two or more correlated receiver operating characteristic curves: a nonparametric approach. Biometrics 1988; 44: 837-45.

27. Pencina MJ, D'Agostino RB Sr, Steyerberg EW. Extensions of net reclassification improvement calculations to measure usefulness of new biomarkers. Stat Med 2011; 30: 11-21.

28. Pencina MJ, D'Agostino RB, D'Agostino RB, Vasan $R S$. Evaluating the added predictive ability of a new marker: from area under the ROC curve to reclassification and beyond. Stat Med 2008; 27: 157-72.

29. Cahalin LP, Mathier MA, Semigran MJ, Dec GW, DiSalvo TG. The six-minute walk test predicts peak oxygen uptake and survival in patients with advanced heart failure. Chest 1996; 110: 325-32.

30. Polkey MI, Spruit MA, Edwards LD, et al. Six-minute-walk test in chronic obstructive pulmonary disease: minimal clinically important difference for death or hospitalization. Am J Respir Crit Care Med 2013; 187: 382-6.

31. Royston P, Altman DG, Sauerbrei W. Dichotomizing continuous predictors in multiple regression: a bad idea. Stat Med 2006; 25: 127-41.

32. Holländer $N$, Sauerbrei $W$, Schumacher $M$. Confidence intervals for the effect of a prognostic factor after selection of an 'optimal' cutpoint. Stat Med 2004; 23: 1701-13.

33. Duceppe E, Patel A, Chan MT, et al. Preoperative N-terminal pro-B-type natriuretic peptide and cardiovascular events after noncardiac surgery: a cohort study. Ann Intern Med 2020; 172 : 96-104.

34. Rothwell PM. Treating individuals 2. Subgroup analysis in randomised controlled trials: importance, indications, and interpretation. Lancet 2005; 365: 176-86.

35. Harrell FE Jr. Regression Modeling Strategies: With Applications to Linear Models, Logistic and Ordinal 
Regression, and Survival Analysis (Springer Series in Statistics). Springer; 2015.

36. Writing Committee for the VISION Study Investigators. Association of postoperative high-sensitivity troponin levels with myocardial injury and 30-day mortality among patients undergoing noncardiac surgery. JAMA 2017; 317: 1642-51.

37. Riley RD, Ensor J, Snell KI, et al. Calculating the sample size required for developing a clinical prediction model. BMJ 2020; DOI: https://doi.org/10.1136/bmj.m441.

38. Fleisher LA, Beckman JA, Brown KA, et al. 2009 ACCF/AHA focused update on perioperative beta blockade incorporated into the ACC/AHA 2007 guidelines on perioperative cardiovascular evaluation and care for noncardiac surgery: a report of the American College of Cardiology Foundation/American Heart Association Task Force On Practice Guidelines. Circulation 2009; 120: e169-276.

39. American Thoracic Society; American College of Chest Physicians. ATS/ACCP statement on cardiopulmonary exercise testing. Am J Respir Crit Care Med 2003; 167: 211-77.

Publisher's Note Springer Nature remains neutral with regard to jurisdictional claims in published maps and institutional affiliations. 